\title{
Correction to: How Does Fat Mass Change in the First Year After Bariatric Surgery? A Systemic Review and Meta-Analysis
}

\author{
Neda Haghighat ${ }^{1}$ - Damoon Ashtary-Larky ${ }^{2} \cdot$ Ladan Aghakhani $^{1} \cdot$ Omid Asbaghi $^{3} \cdot$ Hamidreza Hosseinpour $^{1}$. \\ Babak Hosseini $^{1}$. Ali Shahabinezhad ${ }^{1}$. Arash Pour Mohammad ${ }^{1}$. Seyed Vahid Hosseini ${ }^{1,4}$. Masoud Amini ${ }^{1}$. \\ Cain C.T. Clark ${ }^{5} \cdot$ Alimohammad Bananzadeh $^{1,4}$
}

Published online: 22 December 2021

(C) Springer Science+Business Media, LLC, part of Springer Nature 2021

\section{Obesity Surgery}

https://doi.org/10.1007/s11695-021-05512-9

In the original article the names of the second, fifth, and eighth authors were spelled incorrectly. They are correct here.

Publisher's Note Springer Nature remains neutral with regard to jurisdictional claims in published maps and institutional affiliations.

The online version of the original article can be found at https://doi.org/ 10.1007/s11695-021-05512-9

\footnotetext{
Alimohammad Bananzadeh

bananzadeh@gmail.com

1 Laparoscopy Research Center, Shiraz University of Medical Sciences, Shiraz, Iran

2 Nutrition and Metabolic Diseases Research Center, Ahvaz Jundishapur University of Medical Sciences, Ahvaz, Iran

3 Cancer Research Center, Shahid Beheshti University of Medical Sciences, Tehran, Iran

4 Colorectal Research Center, Shiraz University of Medical Sciences, Shiraz, Iran

5 Center for Intelligent Healthcare, Coventry University, Coventry CV1 5FB, UK
} 